\title{
Downregulated miR-29a promotes B cell overactivation by upregulating Crk-like protein in systemic lupus erythematosus
}

\author{
XINYU SHI $^{1 *}$, LULU YE ${ }^{2 *}$, SHUQI XU ${ }^{3 *}$, GANGQIANG GUO $^{1}$, ZIYI ZUO ${ }^{3}$, MENGKE YE $^{3}$, \\ LEJIANG ZHU ${ }^{4}$, BAOQING LI $^{5}$, XIANGYANG XUE ${ }^{1}$, QIAOAI LIN ${ }^{1}$ and XIAOKAI DING ${ }^{6}$ \\ ${ }^{1}$ Department of Microbiology and Immunology, Institute of Molecular Virology and Immunology,
} Institute of Tropical Medicine, School of Basic Medical Sciences, Wenzhou Medical University, Wenzhou, Zhejiang 325000;

${ }^{2}$ Department of Laboratory Medicine, Anqing Petrochemical Hospital, Anqing, Anhui 246000; ${ }^{3}$ First Clinical College, Wenzhou Medical University; ${ }^{4}$ Department of Nephrology, Wenzhou Central Hospital; ${ }^{5}$ Department of Laboratory Medicine, Second Affiliated Hospital \& Yuying Children's Hospital, Wenzhou Medical University; ${ }^{6}$ Department of Nephrology, First Affiliated Hospital, Wenzhou Medical University, Wenzhou, Zhejiang 325000, P.R. China

Received September 11, 2019; Accepted March 31, 2020

DOI: $10.3892 / \mathrm{mmr} .2020 .11166$

\begin{abstract}
Systemic lupus erythematosus (SLE) is an autoimmune disorder; however, the pathogenesis is not fully understood. Accumulating evidence suggested an important role of microRNAs (miRNA/miR) in autoimmunity. The present study aimed therefore to determine the miRNA expression patterns in the $\mathrm{B}$ cells from the peripheral blood of 66 patients with SLE and 10 healthy controls (HCs) by using an Affymetrix GeneChip ${ }^{\circledR}$ miRNA 2.0 array. In addition, next-generation sequencing was used to obtain the peripheral blood mononuclear cell (PBMC) miRNA profiles from three patients with SLE and three HCs. Candidate miRNAs that were considered to contribute to the pathogenesis of SLE were obtained based on the intersection of miRNA profiles. The analysis revealed a significant downregulation in miR-29a expression levels in B cells from patients with SLE, which was subsequently verified using reverse transcription-quantitative PCR. Based on these results, the expression pattern of miR-29a in SLE was further investigated and its role in the hyperactivity of B cells was determined. miR-29a inhibitors
\end{abstract}

Correspondence to: Professor Qiaoai Lin, Department of Microbiology and Immunology, Institute of Molecular Virology and Immunology, Institute of Tropical Medicine, School of Basic Medical Sciences, Wenzhou Medical University, 22 Qiuzhi Road, Wenzhou, Zhejiang 325000, P.R. China

E-mail: 13957797509@163.com

Dr Xiaokai Ding, Department of Nephrology, First Affiliated Hospital, Wenzhou Medical University, 75 Lucheng Road, Wenzhou, Zhejiang 325000, P.R. China

E-mail: dxk7505@126.com

*Contributed equally

Key words: microRNA-29a, B cell, IgG antibody, Crk-like protein, systemic lupus erythematosus and mimics were transfected into PBMCs obtained from $\mathrm{HCs}$ and patients with SLE, and an ELISA was used to demonstrate that miR-29a inhibition increased the production of $\mathrm{IgG}$. Bioinformatics analysis predicted Crk-like protein (CRKL) as a target gene of miR-29a in patients with SLE. Therefore, CRKL expression levels were compared between patients with SLE and HCs by using western blotting, and its direct transcriptional regulation by miR-29a was determined using a dual-luciferase reporter assay. Low expression levels of miR-29a were revealed to upregulate the expression levels of CRKL in B cells, and the protein expression levels of CRKL in patients with SLE were significantly upregulated compared with the HCs. In conclusion, the results from the present study suggested that miR-29a may affect IgG antibody secretion in $\mathrm{B}$ cells by regulating CRKL, thereby contributing to the development and progression of SLE, which offers a novel candidate target for treatment.

\section{Introduction}

Systemic lupus erythematosus (SLE) is an autoimmune disease with complex clinical manifestations, including nephritis, central nervous system diseases and multi-system organ failure, which can affect multiple organ systems and cause major episodes (1). SLE demonstrates a cyclical disease course, with aggravation and remission occurring alternately (2). SLE is a female sex-biased disease mainly affecting women of childbearing age and with a male:female ratio of 1:9 (3). Although the precise pathogenesis of SLE remains poorly understood, the etiology is considered to be due to a combination of factors, including environmental, genetic, hormonal and immunomodulatory factors (4). Without a clear understanding of the causative agents, the current treatment relies on the alleviation of symptoms with glucocorticoids and non-steroidal anti-inflammatory drugs (5); however, these drugs only help to control the disease progression prophylactically and cannot allow a complete cure. Therefore, the mortality rate of patients with SLE remains high. 
One of the most important characteristics of SLE is the hyperactivity of B cells, which produce various autoantibodies (6). Consequently, autoantibody/autoantigen immune complexes accumulate in various organs and tissues, causing chronic inflammation and tissue damage (7). Therapeutic strategies that regulate B cells are therefore promising. However, the development of effective therapeutic interventions has been hindered by the lack of understanding of $\mathrm{B}$ cell activation underlying mechanisms. Furthermore, the clinical diagnosis of SLE remains a challenge due to the high heterogeneity of the disease (8). Current diagnostic indicators include the SLE Disease Activity Index (SLEDAI) and dsDNA levels (9); however, there is still no gold-standard diagnostic marker. Previous studies have focused on the roles of epigenetic factors in the pathogenesis as potential markers, such as microRNAs (miRNAs/miRs) and circular RNAs $(10,11)$.

miRNAs are a class of non-coding RNAs that regulate gene expression post-transcriptionally $(12,13)$. Previous studies demonstrated that miRNAs serve an important role in the immune system, and that they may potentially serve as disease biomarkers and therapeutic targets $(3,14)$. Several miRNAs have also been proposed to regulate the pathogenesis of autoimmune diseases, such as rheumatoid arthritis and SLE, by affecting B cell function (15). For example, Luo et al (7) reported that decreased expression levels of miR-1246 can enhance the expression levels of early B-cell factor 1 and promote the further activation of $B$ cells in lupus. In addition, miR-29c and miR-29b were also discovered to be two important miRNAs associated with SLE (16). However, to the best of our knowledge, the expression patterns of miR-29a in the $B$ cells of patients with SLE remain unclear.

The miR-29 family includes miR-29a, miR-29b and miR-29c (17) characterized by similar seed sequences, which are expressed in both $\mathrm{T}$ and $\mathrm{B}$ cells $(18,19)$. miR-29b was discovered to induce DNA demethylation and activate the AKT signaling pathway (20), in addition to serving a role in Toll-like receptor inhibition (16). Furthermore, miR-29a was demonstrated to downregulate the expression levels of the Nef protein and interfere with human immunodeficiency virus-1 replication (21). In fact, the deregulation of miRNA expression has been associated with several types of disease and miRNAs have been revealed to function as tumor suppressor genes or oncogenes, depending on their target mRNAs (18). However, the target cells for miR-29a in SLE remain unclear. The present study aimed therefore to investigate the role of miR-29a in B cell hyperactivity and to determine its contribution to the pathogenesis of SLE. miR-29a may thus be considered as a potential diagnostic marker or therapeutic target in SLE.

\section{Materials and methods}

Patient studies. The present study was approved by the Medical and Ethical Committees of Wenzhou Medical University (Zhejiang, China) and informed written consent was obtained from all participants. The study included a total of 106 patients with SLE (15 males, 91 females, age range, 28-52 years; mean age, $39.75 \pm 10.00$ years) who were admitted to the Department of Rheumatology and Nephrology, First Affiliated Hospital of Wenzhou Medical University between February 2018 and October 2018. All patients with SLE fulfilled the SLE Classification Criteria of the American College of Rheumatology (22). The SLEDAI was evaluated according to the systemic symptoms prior to blood collection (23). A total of 3 patients with SLE with an SLEDAI score $>10$ were selected for miRNA-Seq and 66 patients with SLE with an SLEDAI score $>10$ were selected for miRNA hybridization chip analysis (Table SI). Another 37 patients with SLE were randomly selected for validation. A total of 43 healthy individuals ( 10 males, 33 females, age range, $26-50$ years; mean age, $38 \pm 12$ years) were also admitted to the First Affiliated Hospital of Wenzhou Medical University between February 2018 and October 2018, who did not have arthralgia, heart or renal failure, and had not been diagnosed with an autoimmune disease served as healthy controls (HCs). Whole blood samples $(5 \mathrm{ml})$ were collected from all participants into EDTA-coated tubes after obtaining informed written consent.

Peripheral blood mononuclear cell (PBMC) isolation and RNA sequencing. PBMCs were isolated within $4 \mathrm{~h}$ of blood sample collection using human peripheral blood lymphocyte separation medium (3) (Tianjin HaoYang Biological Manufacture Co., Ltd.). RNA extraction from PBMC and RNA sequencing were performed as previously reported (3).

$B$ cell isolation and miRNA hybridization chip detection. B cells $\left(1 \times 10^{6}\right)$ from PBMCs were labeled with anti-human CD19 magnetic particles (cat. no. 130-050-301; Miltenyi Biotec $\mathrm{GmbH}$ ), according to the manufacturer protocol, and obtained by passing the sample through a magnetic stand. The positive fraction was further analyzed using flow cytometry.

Total RNA was extracted from the B cells $(1-2 \mu \mathrm{g})$ as previously described (3). The NanoDrop ND-1000 instrument (Thermo Fisher Scientific, Inc.) was used to measure total RNA concentration. The OD260/OD280 value was used as an index of RNA purity. If the OD260/OD280 value range is $1.8-2.1$, the RNA purity is acceptable. The $200 \mathrm{ng}$ RNA samples were then loaded onto an Affymetrix miRNA 2.0 array chip (Affymetrix; Thermo Fisher Scientific, Inc.); the chip uses Sanger miRBase version 15.0 (genewiz.com.cn/)sequences with a probe length of 25 bases and a design covering 20,162 miRNAs. Poly(A) polymerase was used to add poly(A) tails to the miRNA samples and the miRNAs were fluorescently labeled and purified using the FlashTag Biotin HSR Ligation Labelling kit (Applied Biosystems; Thermo Fisher Scientific, Inc.) according to the manufacturer's protocol. The hybridization solution was configured according to the manufacturer's instructions (Applied Biosystems; Thermo Fisher Scientific, Inc.), injected into the chip and the sample port was sealed with a sticker. The chip was then placed in a hybridization oven and hybridized at $60 \mathrm{xg}$ for $16 \mathrm{~h}$ at $48^{\circ} \mathrm{C}$. The hybridization solution was subsequently aspirated with a pipette tip and scanned after elution using an Affymetrix 7G/TG scanner (Affymetrix; Thermo Fisher Scientific, Inc.). The image was converted into a digital signal based on the fluorescence intensity. Affymetrix miRNA QC Tool version 1.1.1.0 (Thermo Fisher Scientific, Inc.) was used for data summarization and initial quality control.

Reverse transcription-quantitative PCR (RT-qPCR). Total RNA was extracted from B cells as previously described (3). 
Table I. Upregulated miRNA expression profiles in B cells of patients with SLE and HCs determined using a miRNA hybridization chip.

\begin{tabular}{lc} 
miRNA & Fold-change $^{\text {a }}(\mathrm{SLE} / \mathrm{HC})$ \\
\hline hsa-miR-2054 & 18.29 \\
hsa-miR-1224-3p & 7.44 \\
hsa-miR-1249 & 6.09 \\
hsa-miR-2116-star & 4.30 \\
hsa-miR-4258 & 4.12 \\
hsa-miR-4313 & 3.07 \\
hsa-miR-1915 & 2.40 \\
hsa-miR-220b & 2.13 \\
hsa-miR-19b & 2.23 \\
hsa-miR-15b & 2.30 \\
hsa-miR-22 & 2.37 \\
hsa-miR-155 & 2.11 \\
\hline
\end{tabular}

${ }^{\text {aF }}$ old-change $>2$. miRNA/miR, microRNA; SLE, systemic lupus erythematosus; HC, healthy control.

A volume of $1 \mu \mathrm{g}$ RNA was reverse transcribed into cDNA according to the manufacturer's protocol of ReverTra Ace ${ }^{\circledR} \mathrm{qPCR}$ RT Master mix (Toyobo Life Science). qPCR was performed using $1 \mu \mathrm{g}$ cDNA as a template and a QuantiNova SYBR Green PCR kit (Qiagen,GmbH) and miR-29a/U6-specific stem-loop primers. The following primers were used: miR-29a: Forward, 5'-GCGGGACTGATTTCTTTTGG-3' and reverse, 5'-GTG CAGGGTCCGAGGTATTC-3' and U6: Forward, 5'-GTGCTC GCTTCGGCAGCACAT-3' and reverse, 5'-TACCTTGCGAAG TGCTTAAAC-3'. Samples were run in triplicate in 96-well plates using an Applied Biosystems 7900HT Real-Time PCR thermocycler (Applied Biosystems; Thermo Fisher Scientific, Inc.). Preamplification was performed at $95^{\circ} \mathrm{C}$ for $2 \mathrm{~min}$, denaturation at $95^{\circ} \mathrm{C}$ for $15 \mathrm{sec}$, annealing and extension at $60^{\circ} \mathrm{C}$ for $1 \mathrm{~min}, 40$ cycles. Expression levels were analyzed using the $2^{-\Delta \Delta \mathrm{Ct}}$ method (24) and normalized to U6 as the reference gene.

Cell culture and transfection. Raji cells (Chinese Academy of Sciences Shanghai Cell Bank) were cultured in RPMI-1640 medium (Gibco; Thermo Fisher Scientific, Inc.), supplemented with $10 \%$ FBS (GE Healthcare Bio-Sciences) in a $37^{\circ} \mathrm{C}$ incubator under a humidified atmosphere containing $5 \% \mathrm{CO}_{2}$. Raji cells were subsequently plated into a 6-well cell culture plate at a density of $1 \times 10^{6}$ cells/well and $300 \mu \mathrm{l}$ electroporation solution (BTX, Genetronics, Inc.) was added to each well. Subsequently, $60 \mathrm{nM}$ of miR-29a-mimic (5'-UAGCACCAU CUGAAAUCGGUUA-3'), mimic-NC (5'-UUCUCCGAA CGUGUCACGUTT-3'), miR-29a-inhibitor (5'-UAACCGAUU UCAGAUGGUGCUA-3') or inhibitor-NC (5'-CAGUACUUU UGUGUAGUACAA-3') (GenePharma, Shanghai, China) were added to the solution, which were all FAM-conjugated. The control group (electrotransfected without transfection components) and the blank group (not electrotransfected) were also set up. The mixed cell suspension was transferred to the electroporation cup and an ECM 830 Square Wave electroporator
(BTX, Genetronics, Inc.) was used for the electroporation transfection $(250 \mathrm{~V} ; 10 \mathrm{~ms})$ at $37^{\circ} \mathrm{C}$ for $10 \mathrm{~min}$. Following transfection, cells were cultured in RPMI-1640 medium containing $10 \%$ FBS. The experiment was subsequently divided into two groups as follows: The first group of cells was used to evaluate the transfection efficiency and the second group of cells was used for subsequent experiments. After $48 \mathrm{~h}$ of incubation, the first group of collected cells was used to evaluate the transfection efficiency by flow cytometry and the second group of cells and supernatant were collected and stored at $-20^{\circ} \mathrm{C}$ for further experimentation.

Flow cytometry. Following $48 \mathrm{~h}$ transfection, the cell suspension was centrifuged for $5 \mathrm{~min}$ at $1,000 \mathrm{x}$ at $4{ }^{\circ} \mathrm{C}$; the supernatant was discarded and the cell pellet was resuspended in PBS at a cell density of $1 \times 10^{6}$ cells $/ \mathrm{ml}$ for analysis using a BD Accuri ${ }^{\mathrm{TM}}$ C6 Flow Cytometer (BD Biosciences). The control group was used as a negative control to set the gates for the flow cytometry. The transfection efficiency of the miR-29a-mimic, mimic-NC, miR-29a-inhibitor and inhibitor-NC was subsequently analyzed to confirm that all transfections were effective. Data was analyzed using BD_Accuri_C6 software 2.0 (BD Biosciences).

IgG ELISAs. At $48 \mathrm{~h}$ post-transfection, the cell suspension was centrifuged for $10 \mathrm{~min}$ at $12,000 \mathrm{x} \mathrm{g}$ at $4^{\circ} \mathrm{C}$. The $\mathrm{IgG}$ concentration in the $\mathrm{B}$ cell culture supernatant was determined using a human IgG ELISA kit (cat. no. ab100547; Abcam), according to the manufacturer's protocol. All experiments were performed in triplicate. The absorbance of the sample was read at $450 \mathrm{~nm}$ using a fully automated ELISA processor (Roche Applied Science).

Target gene prediction and bioinformatics analysis of miRNAs and mRNAs. A total of four online databases were used to predict the target genes of miRNAs: miRanda (http://www. microrna.org/), PolymiRTS database (http://compbio.uthsc. edu/miRSNP/home.php), TargetMiner (https://www.isical. ac.in/ Bioinfo_miu/targetminer20.htm) and CLIP-Seq (http://mirtarclip.mbc.nctu.edu.Tw/). Venny 2.1.0 software was used to draw the Venn diagrams to obtain the intersection of the prediction results from the four databases. The 3 ' untranslated region (UTR) sequence of the target gene was obtained using TableBrowser (http://genome.ucsc.edu/cgi-bin/hgTables). The secondary structure of the mRNA and miRNA interactions was predicted using RNAhybrid (bibiserv.cebitec.uni-bielefeld. de/rnahybrid/) (3). Target genes associated with B cell function or IgG levels were identified using Chilibot (chilibot.net).

Dual-luciferase reporter assay. The 3' UTR of CRKL, which contains the predicted target site for miR-29a, was cloned into the pmirGLO luciferase reporter vector. $293 \mathrm{~T}$ cells $\left(1 \times 10^{6}\right)$ were co-transfected (in 24-well plates with $1 \mu \mathrm{g}$ firefly luciferase reporter vector (pmirGLO-CRKL-3UTR) or control vector (pmirGLO-CRKL -3UTR-mut; cat. no. E1910Promega Corporation) containing Renilla luciferase and $60 \mathrm{nM}$ miR-29a-mimic or mimic-NC. Following incubation at $37^{\circ} \mathrm{C}$ for $48 \mathrm{~h}$, the firefly and Renilla luciferase activities were detected using a Dual-Luciferase Reporter assay system (Promega Corporation), according to the manufacturer's 
Table II. miRNA expression level profiles in B cells of patients with SLE and HCs determined using a miRNA hybridization chip.

\begin{tabular}{|c|c|}
\hline miRNA & Fold-change $^{\mathrm{a}}(\mathrm{SLE} / \mathrm{HC})$ \\
\hline hsa-miR-29a & 0.03 \\
\hline hsa-miR-26a & 0.03 \\
\hline hsa-miR-150 & 0.03 \\
\hline hsa-miR-103 & 0.04 \\
\hline hsa-let-7b & 0.04 \\
\hline hsa-miR-342-3p & 0.04 \\
\hline hsa-miR-16 & 0.05 \\
\hline hsa-miR-1826 & 0.05 \\
\hline hsa-miR-191 & 0.06 \\
\hline hsa-miR-107 & 0.06 \\
\hline hsa-miR-24 & 0.06 \\
\hline hsa-miR-92a & 0.07 \\
\hline hsa-let-7a & 0.08 \\
\hline hsa-let-7d & 0.09 \\
\hline hsa-miR-181a & 0.09 \\
\hline hsa-miR-23a & 0.10 \\
\hline hsa-miR-20a & 0.11 \\
\hline hsa-miR-320a & 0.12 \\
\hline hsa-miR-17 & 0.12 \\
\hline hsa-let-7i & 0.12 \\
\hline hsa-miR-140-3p & 0.13 \\
\hline hsa-miR-222 & 0.13 \\
\hline hsa-miR-106a & 0.15 \\
\hline hsa-miR-221 & 0.15 \\
\hline hsa-miR-320c & 0.15 \\
\hline hsa-miR-320b & 0.15 \\
\hline hsa-let-7c & 0.16 \\
\hline hsa-miR-93 & 0.17 \\
\hline hsa-miR-29 & 0.17 \\
\hline hsa-miR-185 & 0.19 \\
\hline hsa-let-7g & 0.23 \\
\hline hsa-miR-106b & 0.25 \\
\hline hsa-miR-146a & 0.25 \\
\hline hsa-miR-425 & 0.28 \\
\hline hsa-miR-23b & 0.29 \\
\hline hsa-miR-27a & 0.30 \\
\hline hsa-miR-361-5p & 0.30 \\
\hline hsa-miR-151-5p & 0.30 \\
\hline hsa-miR-126 & 0.47 \\
\hline hsa-miR-320d & 0.29 \\
\hline hsa-miR-1975 & 0.21 \\
\hline hsa-let-7f & 0.37 \\
\hline hsa-miR-652 & 0.37 \\
\hline hsa-miR-4298 & 0.39 \\
\hline hsa-miR-638 & 0.29 \\
\hline hsa-miR-181b & 0.33 \\
\hline hsa-miR-20b & 0.39 \\
\hline hsa-miR-494 & 0.47 \\
\hline hsa-miR-4270 & 0.32 \\
\hline hsa-miR-223 & 0.48 \\
\hline
\end{tabular}

Table II. Continued.

\begin{tabular}{lc}
\hline miRNA & Fold-change $^{\mathrm{a}}$ (SLE/HC) \\
\hline hsa-miR-149-star & 0.32 \\
hsa-miR-762 & 0.28 \\
hsa-miR-25 & 0.38 \\
hsa-miR-378 & 0.34
\end{tabular}

${ }^{\text {aFold-change }}>2$. miRNA/miR, microRNA; SLE, systemic lupus erythematosus; HC, healthy control.

protocol. Firefly luciferase activity was normalized to Renilla luciferase activity.

Western blotting. Total protein was extracted from Raji cells and PBMCs using RIPA lysis buffer (Beijing Solarbio Science \& Technology Co.) supplemented with protease inhibitor cocktail (Pierce; Thermo Fisher Scientific, Inc.). A BCA protein assay kit (Thermo Fisher Scientific, Inc.) was used to determine the protein concentration. A total of $15 \mu \mathrm{g}$ protein was separated by $12 \%$ SDS-PAGE. Proteins were subsequently transferred onto PVDF membranes (EMD Millipore) and blocked in 5\% skim milk at $37^{\circ} \mathrm{C}$ for $1.5 \mathrm{~h}$. The membranes were incubated with a monoclonal rabbit anti-CRKL (1:1,000; cat. no. ab32126; Abcam) and monoclonal mouse anti-GAPDH primary antibody (1:1,000; cat. no. AB-M-M001; Xianzhi Biotechnology Company) overnight at $4{ }^{\circ} \mathrm{C}$. Following the primary antibody incubation, the membranes were washed five times for $5 \mathrm{~min}$ each with TBST $(0.05 \%$ Tween-20) buffer and then incubated with a horseradish peroxidase (HRP)-conjugated goat anti-rabbit IgG secondary antibody(1:5,000; Hangzhou Multi Sciences) and HRP-conjugated goat anti-mouse IgG secondary antibody (1:5,000; Hangzhou Multi Sciences) for $1.5 \mathrm{~h}$ at $37^{\circ} \mathrm{C}$. Protein bands were detected using a chemiluminescence solution (Bio-Rad Laboratories, Inc.) and visualized using a Gel Doc 2000 ChemiDoc imaging system (Bio-Rad Laboratories, Inc.). Relative expression level of CRKL was normalized to endogenous control GAPDH using ImageJ software v1.8.0 (National Institutes of Health).

Statistical analysis. Statistical analysis was performed using SPSS 22.0 software (IBM Corp.); expression levels obtained following RT-qPCR data were analyzed using GraphPad Prism software (version 5.0; GraphPad Software, Inc.). The data were presented as the mean \pm standard deviation of three repeats. An independent Student's t-test was used to determine the statistical differences between two groups, and multigroup comparisons of the means were performed via one-way ANOVA with post hoc Student-Newman-Keuls test. Sensitivity and specificity values were determined using a receiving operating characteristic (ROC) curve. $\mathrm{P}<0.05$ was considered to indicate a statistically significant difference.

\section{Results}

Expression profiles of B cell miRNAs from patients with SLE and HCs. The miRNA array revealed that compared with HCs, 
A

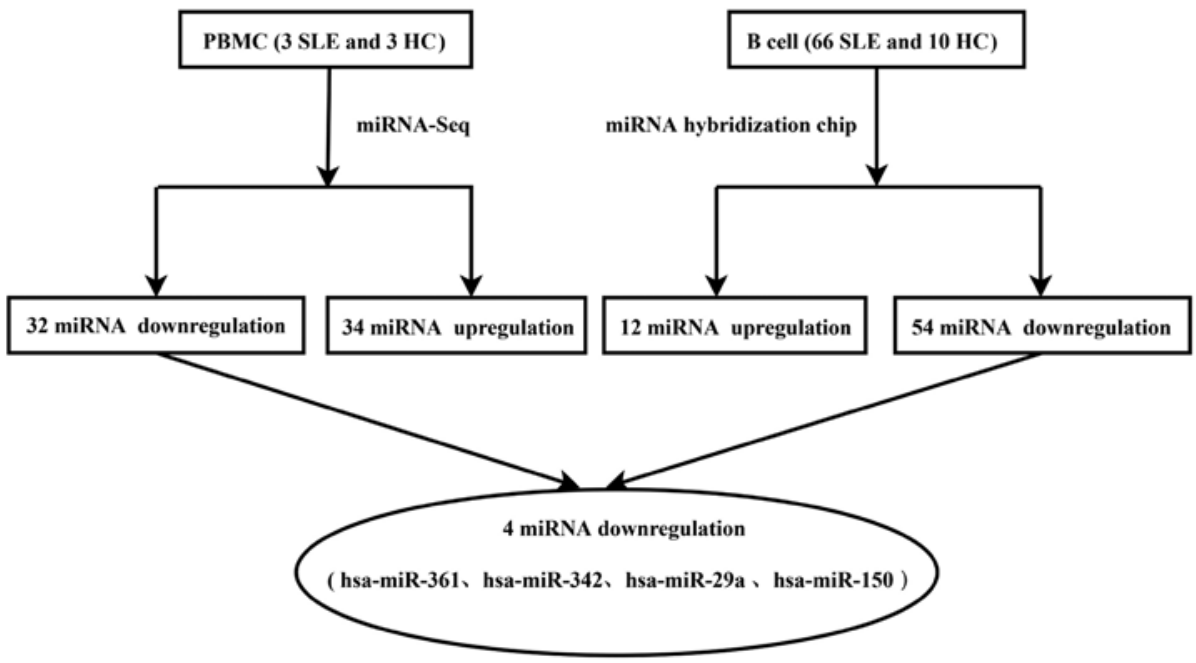

B

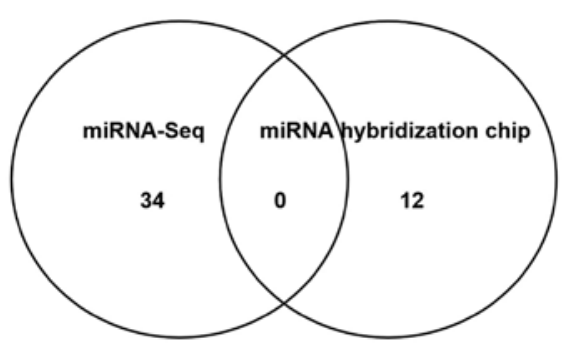

C

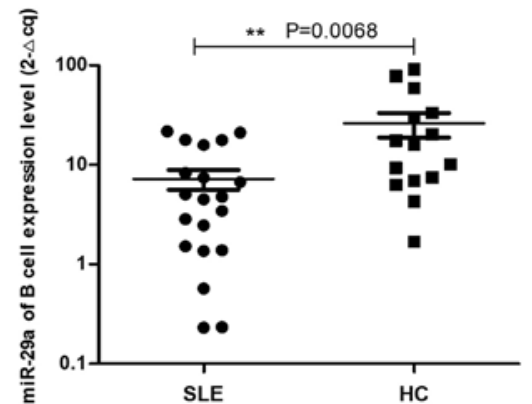

Downregulation

D

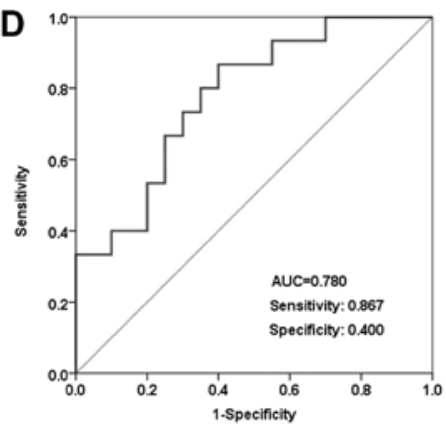

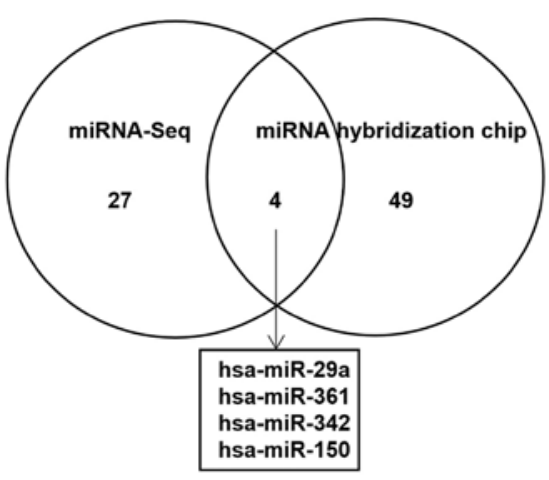

Figure 1. Expression profiles of miRNAs between patients with SLE and HCs. (A) Flowchart for the expression profiles of B cell miRNAs obtained from the miRNA hybridization chip and miRNA-Seq analysis in patients with SLE and HCs. (B) Venn diagram depicting the analysis of the commonly upregulated (left) and downregulated (right) miRNAs between patients with SLE and HCs, as determined by miRNA-Seq and miRNA hybridization chip analysis (C) miR-29a expression levels in B cells from patients with SLE ( $\mathrm{n}=20 ; 3$ males, 17 females) were significantly downregulated compared with the HCs ( $\mathrm{n}=15$; 8 males, 7 females). The arbitrary value was amplified 100-fold. ${ }^{* *} \mathrm{P}<0.01$ (D) Clinical diagnostic value of miR-29a in B cells from patients with SLE and HCs was analyzed using receiving operating characteristic curves. miRNA/miR, microRNA; SLE, systemic lupus erythematosus; HC, healthy control; AUC, area under the curve.

there were 66 miRNAs in patients with active SLE (SLEDAI $\geq 10$ ), including 54 miRNAs that were downregulated and 12 miRNAs that were upregulated in patients with SLE (Tables I and II; Fig. 1A). In addition, the expression profiles of miRNAs in the PBMCs from three patients with SLE and three HCs confirmed the significant differential expression of these 66 miRNAs $(\mathrm{P}<0.05$ and fold change $>2)$, whereby 34 upregulated and 32 downregulated miRNAs were discovered in the PBMCs of patients with SLE. The differential expression of miRNAs in the PBMCs of patients with SLE and HCs is presented as a heatmap and cluster diagram in Fig. S1A, whereas the combined analysis of miRNA hybridization chips and miRNA-Seq data is presented as a flow chart in Fig. 1A. In the intersection data of these two analyses, miR-29a, miR-361, miR-342 and miR-150 were all found to be commonly downregulated in patients with SLE (Fig. 1B). Therefore, the present study focused on the role of miR-29a in the hyperactivity of B cells in SLE.

miR-29a expression levels are significantly downregulated in B cells from patients with SLE. Flow cytometry analysis 

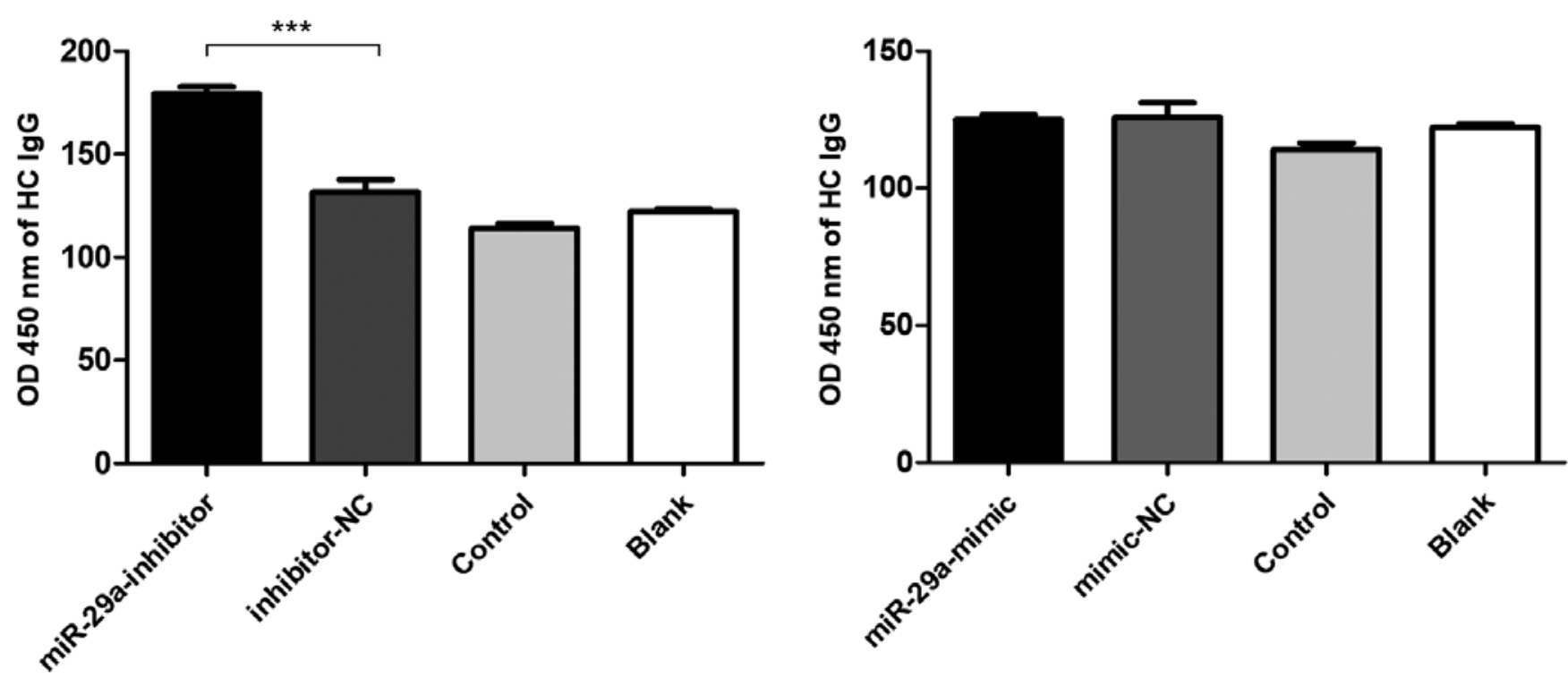

Figure 2. Downregulated miR-29a promotes CRKL protein expression levels. Levels of total IgG were significantly increased following the electrotransfection of the miR-29a inhibitor into the peripheral blood mononuclear cells of $\mathrm{HCs}$. ${ }^{* * *} \mathrm{P}<0.001$. SLE, systemic lupus erythematosus; HC, healthy control; miR, microRNA; NC, negative control.

demonstrated that the pure B cells of patients with $\operatorname{SLE}(n=20)$ and $\mathrm{HCs}(\mathrm{n}=10)$ accounted for $85.9 \%$ of the total number of cells according to the scatter signal (Fig. S2A). Furthermore, the RT-qPCR data revealed that miR-29a expression level was significantly downregulated in B cells from patients with SLE compared with HCs (Fig. 1C). The area under the ROC curve value was 0.780 , with a sensitivity of 0.867 and a specificity of 0.400 (Fig. 1D). These results suggested that miR-29a may be considered as a novel diagnostic marker for SLE prognosis.

Low miR-29a expression levels promote IgG production. Based on the fluorescence analysis of FAM expression, the transfection efficiency of the miR-29a mimic, mimic-NC, miR-29a inhibitor and inhibitor-NC in PBMCs was 41.2, 47.8, 59.8 and $65.4 \%$, respectively (Fig. S2B). In addition, the levels of total IgG in the supernatant were significantly increased following the transfection of the miR-29a inhibitor into the PBMCs obtained from HCs (Fig. 2).

miR-29a directly targets $C R K L$. The MiRanda, PolymiRTS Database, TargetMiner and CLIP-Seq databases predicted $1,565,32,1,756$ and 267 target genes for miR-29a, respectively (Fig. 3A). After taking the intersection of these results, two predicted target genes were obtained: $C R K L$ and Rasrelated GTP-binding protein $\mathrm{C}(R R A G C)$. The target position and secondary structure of the mir-29a-3p interactions with $C R K L$ were subsequently predicted (Fig. 3B). These findings were further supported by the Chilibot database analysis, which predicted $C R K L$ to be associated with both B cell function and IgG levels $(21,22)$. The results showed that there are currently 18 reports that CRKL is associated with $\mathrm{B}$ cells, and four reports that CRKL is associated with IgG production. (Fig. 3C).

The dual-luciferase reporter assay revealed that the direct binding of miR-29a to the pmirGLO-CRKL-3'-UTR transcript significantly repressed the relative luciferase activity compared with the direct binding of mimic-NC to the pmirGLO-CRKL-3'UTR transcript ( $\mathrm{P}<0.01$; Fig. 3D), which further suggested that CRKL may be a direct downstream target of miR-29a. In addition, mutations in the miR-29a-binding region within the 3'-UTR of CRKL abolished the decrease observed in the relative luciferase activity between the mimic-NC- and miR-29a-transfected groups, indicating the specificity of the target site of CRKL (Fig. 3D). Next, western blotting was performed to determine whether miR-29a downregulates the protein expression level of CRKL. CRKL protein levels were significantly decreased in Raji cells transfected with miR-29a mimic compared with levels in cells transfected with the mimic-NC. CRKL protein levels were significantly increased in Raji cells transfected with miR-29a inhibitor compared with levels in cells transfected with the inhibitor-NC (Fig. 3E). Furthermore, western blotting analysis of the B cells of patients with SLE $(n=7)$ and HCs $(n=5)$ demonstrated a significant increase in the expression levels of the CRKL protein in the B cells of patients with SLE compared with HCs (Fig. 3F).

\section{Discussion}

SLE is a systemic autoimmune disease caused by a wide range of defects in the immune regulation $(25,26)$, including the overactivation of B cell as one of the prominent features (26). For example, Sherer et al (26) proposed that SLE is caused by multiple genetic defects, resulting in the excessive activation of B cells. In addition, Zhao et al (27) reported that the intricate clinical manifestations of SLE may be immune-based owing to the loss of autoantibodies and autotolerance produced by the $\mathrm{B}$ cells.

Several independent analyses of miRNA expression levels have demonstrated marked differences in the miRNA profiles between patients with SLE and HCs (7), which provided 
A

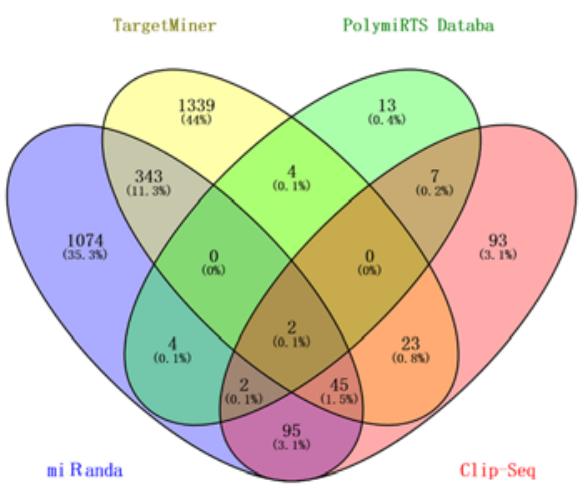

C
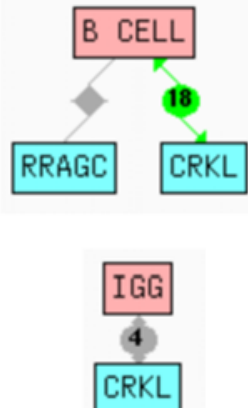

B

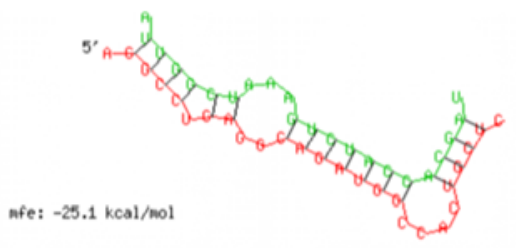

\section{CRKL-3'UTR 5'AGGCCUGAGG CAGAUGGCCACUGCUC hsa-miR--29a-3p 3 ' AUUGG-CUAAAGUCUACC- ACGAU}

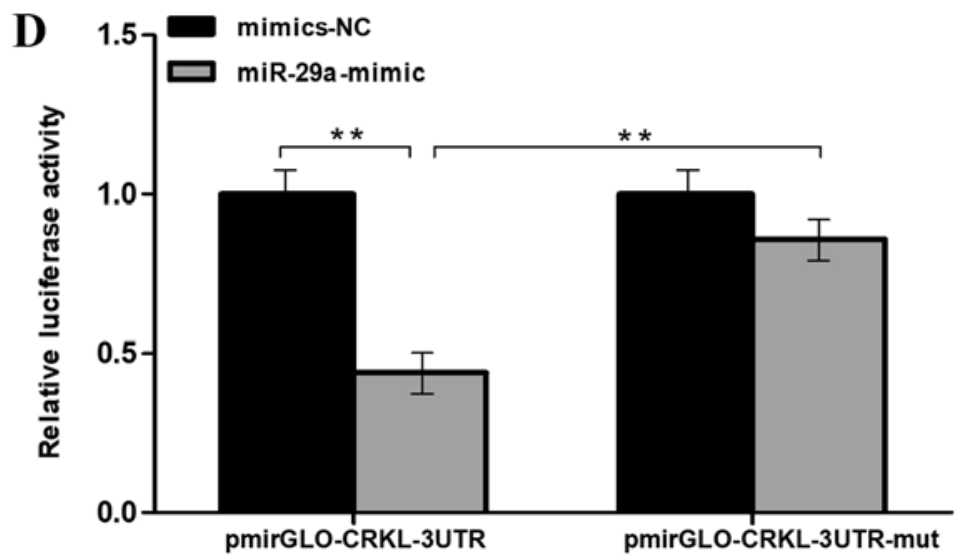

E

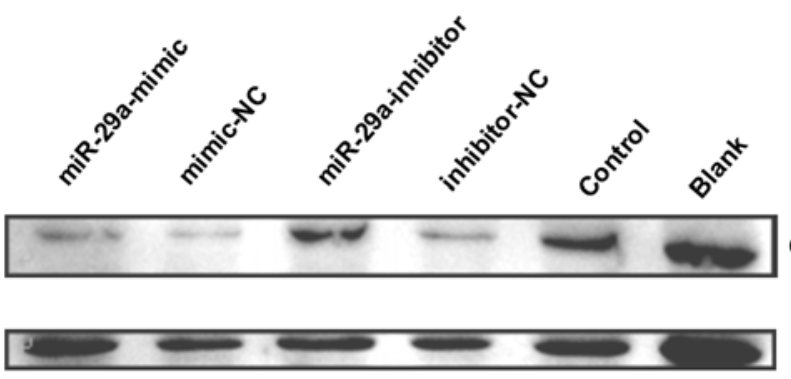

CRKL

$\beta$-actin

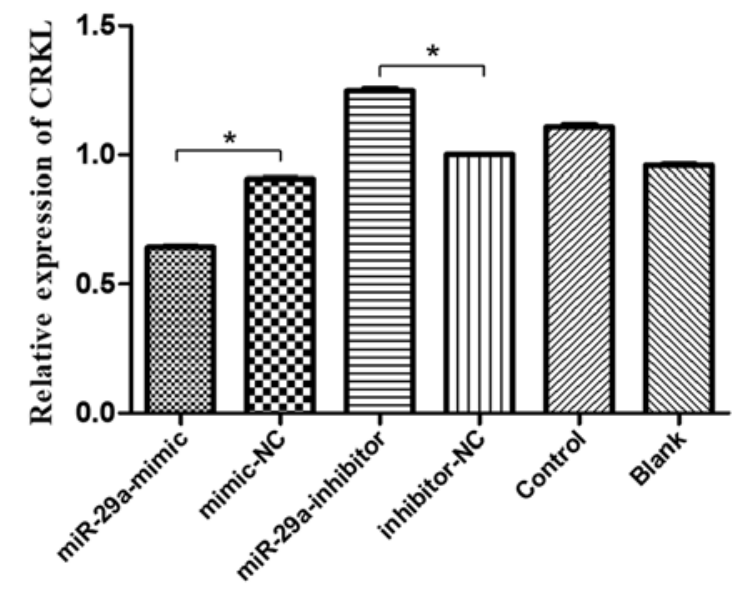

$\mathbf{F}$
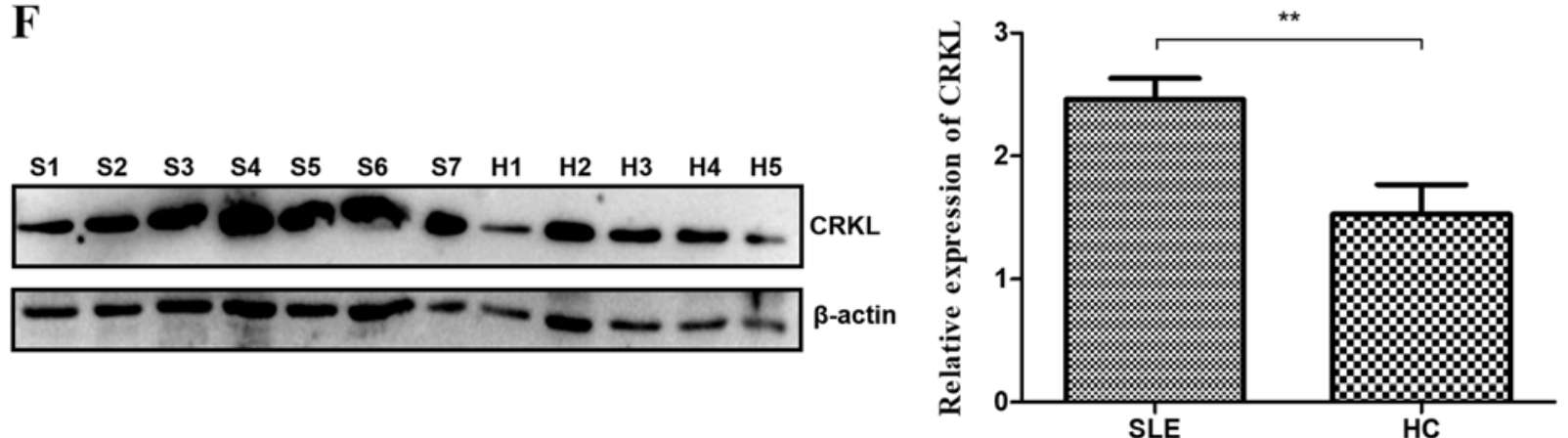

Figure 3. Predicted miR-29a target genes and confirmation. (A) Intersection of predicted target genes for miR-29a-3p. The number of target genes of miR-29a-3p was predicted by four databases to be 1565, 32, 1756 and 267. A total of two predicted target genes were obtained as the intersection. (B) Target position and secondary structure of the miR-29a-3p interaction with $C R K L$. (C) The target gene $(C R K L)$ functions associated with B cell or IgG level regulation were identified. (D) Dual-luciferase reporter assay was used to determine that the direct binding of miR-29a to the pmirGLO-CRKL-3'-UTR transcript repressed the luciferase activity. (E) Western blotting was used to analyze the expression levels of the CRKL protein. Raji cells were transfected with a miR-29a-mimic, mimic-NC, miR-29a-inhibitor or inhibitor-NC. All values are presented as the mean \pm SD of three replicates. (F) Western blotting was used to analyze the expression levels of CRKL protein in the B cells of seven patients with SLE and five HCs. All values are presented as the mean $\pm \mathrm{SD}$ of three repeats. ${ }^{*} \mathrm{P}<0.05$ vs. mimic-NC or inhibitor- NC group; ${ }^{* *} \mathrm{P}<0.01$. SLE/S, systemic lupus erythematosus; $\mathrm{HC} / \mathrm{H}$, healthy control; miR, microRNA; NC, negative control; CRKL, Crk-like protein; UTR, untranslated region; mut, mutant; RRAGC, Ras-related GTP-binding protein C. 
important information for investigating the pathogenesis of SLE, and for developing novel diagnostic biomarkers and treatment targets (7). The present study demonstrated that the miR-29a expression levels in the B cells of patients with SLE were significantly decreased compared with those in HCs. Therefore, it was hypothesized that miR-29a expression level may be used as a potential biomarker and a novel therapeutic target for the treatment of active SLE.

miR-29a expression level has also been reported to be significantly reduced in certain cancers, including neuroblastoma (23), osteosarcoma (19) and mantle cell lymphoma (28), suggesting that miR-29a may be a diagnostic and prognostic biomarker for these different diseases. For example, Maugeri et al (23) reported that miR29a-3p expression level was downregulated in vivo in a mouse neuroblastoma progression model, suggesting a new therapeutic target for neuroblastoma. However, Liu et al (29) demonstrated that the expression level of miR-29a in non-small cell lung cancer (NSCLC) tissues was increased compared with the normal lung tissues, whereas the expression level of the metastasis suppressor protein 1 (MTSS1) was significantly decreased compared with in the normal lung tissues. The study further hypothesized that miR-29a might therefore downregulate MTSS1 protein expression level, thereby promoting the proliferation and invasion of NSCLC cells (29). These studies indicated that miR-29a may have different regulatory roles in different cell lines; however, to the best of our knowledge, there has been little research conducted to date on the role of miR-29a in SLE. In the present study, a bioinformatics approach was used to determine the predicted target genes of miR-29a from four miRNA target gene prediction sites. After taking the intersection, the candidate gene CRKL was chosen as the target. Using western blotting and ELISA assays, the downregulation of miR-29a expression levels in B cells obtained from patients with SLE was suggested to enhance B cell reactivity through the witnessed upregulation of the expression levels of its target gene, CRKL. The dual-luciferase reporter assay further validated the presence of miR-29a-binding sites in the 3'UTR of CRKL.

CRKL encodes the CRK-like protooncogene adaptor protein, and the function of CRKL has been discovered to be associated with the regulation of B cells (30) and IgG levels (31). However, to the best of our knowledge, there are only a few reports detailing the involvement of CRKL in the pathogenesis of SLE. In our previous study, high-throughput sequencing analysis of RNAs from PBMCs of three patients with SLE and three HCs demonstrated that the expression levels of CRKL were upregulated in patients with SLE (3). The present study demonstrated that the expression level of the CRKL protein in B cells from patients with SLE was significantly increased compared with those in the B cells from HCs.

In conclusion, the findings from the present study suggested that the decreased expression levels of miR-29a in patients with SLE may promote the expression of its downstream target CRKL to increase the IgG secretion by B cells. Therefore, therapeutic strategies to restore the normal expression levels of miR-29a may serve as a potential method for the effective treatment of SLE.

\section{Acknowledgements}

Not applicable.

\section{Funding}

The present study was supported by the Zhejiang Provincial Natural Science Foundation of China (grant no. LY12H05003), the Zhejiang Science and Technology Bureau (grant no. 2016C33214), the Medical Health Science and Technology Project of Zhejiang Provincial Health Commission (grant no. 2019K Y453), the Zhejiang University Student New Talents Program (grant no. 2018R413016) and the Wenzhou Municipal Science and Technology Bureau (grant no. Y20160018).

\section{Availability of data and materials}

The datasets used and/or analyzed during the current study are available from the corresponding author on reasonable request.

\section{Authors' contributions}

XS, LY and SX contributed to the design and planning of the experiments; XS and LY wrote the manuscript; XS, LY, SX, GG, ZZ and MY performed the laboratory experiments; LZ, $\mathrm{BL}$ and $\mathrm{XX}$ conducted experiments and provided material support; QL and XD analyzed the data and revised the manuscript. All authors read and approved the final manuscript.

\section{Ethics approval and consent to participate}

This study is performed in accordance with ethical standards. The study was approved by the Medical and Ethical Committees of the Wenzhou Medical University (Zhejiang, China). All participants provided written informed consent.

\section{Patient consent for publication}

Not applicable.

\section{Competing interests}

The authors declare that they have no competing interests.

\section{References}

1. Frieri M: Mechanisms of disease for the clinician: Systemic lupus erythematosus. Ann Allerg Asthma Im 110: 228-232. 2013.

2. Ziegelasch M, van Delft MA, Wallin P, Skogh T, Magro-Checa C, Steup-Beekman GM, Trouw LA, Kastbom A and Sjöwall C: Antibodies against carbamylated proteins and cyclic citrullinated peptides in systemic lupus erythematosus: Results from two well-defined European cohorts. Arthritis Res Ther 18: 289, 2016.

3. Guo G, Wang H, Shi X, Ye L, Wu K, Lin K, Ye S, Li B, Zhang H, Lin Q, et al: NovelmiRNA-25 inhibits AMPD2 in peripheral blood mononuclear cells of patients with systemic lupus erythematosus and represents a promising novel biomarker. J Transl Med 16: 370, 2018.

4. Jeltsch-David H and Muller S: Neuropsychiatric systemic lupus erythematosus: Pathogenesis and biomarkers. Nat Rev Neurol 10: 579-596, 2014. 
5. Bernatsky S, Joseph L, Boivin JF, Gordon C, Urowitz M, Gladman D, Fortin PR, Ginzler E, Bae SC, Barr S, et al: The relationship between cancer and medication exposures in systemic lupus erythaematosus: A case-cohort study. Ann Rheum Dis 67: 74-79, 2008.

6. Baudino L, Yoshinobu K, Morito N, Santiago-Raber ML and Izui S: Role of endogenous retroviruses in murine SLE. Autoimmun Rev 10: 27-34, 2010.

7. Luo S, Liu Y, Liang G, Zhao M, Wu H, Liang Y, Qiu X, Tan Y, Dai Y, Yung S, et al: The role of microRNA-1246 in the regulation of B cell activation and the pathogenesis of systemic lupus erythematosus. Clin Epigenetics 7: 24, 2015.

8. Yu F, Haas M, Glassock R and Zhao MH: Redefining lupus nephritis: Clinical implications of pathophysiologic subtypes. Nat Rev Nephrol 13: 483-495, 2017.

9. Xie HH, Shen H, Zhang L, Cui MY, Xia LP and Lu J: Elevated serum interleukin-34 level in patients with systemic lupus erythematosus is associated with disease activity. Sci Rep 8 : 3462, 2018.

10. Zhou B, Zuo XX, Li YS, Gao SM, Dai XD, Zhu HL and Luo H: Integration of microRNA and mRNA expression profiles in the skin of systemic sclerosis patients. Sci Rep 7: 42899, 2017.

11. Luo Q, Liu J, Fu B, Zhang L, Guo Y, Huang Z and Li J: Circular RNAs Hsa_circ_0002715 and Hsa_circ_0035197 in peripheral blood are novel potential biomarkers for new-onset rheumatoid arthritis. Dis Markers 2019: 2073139, 2019.

12. He L and Hannon GJ: MicroRNAs: Small RNAs with a big role in gene regulation. Nat Rev Genet 5: 522-531, 2004.

13. Xiao C and Rajewsky K: microrna control in the immune system: Basic principles. Cell 136: 26-36, 2009.

14. Ma X and Liu Q: MicroRNAs in the pathogenesis of systemic lupus erythematosus. Int J Rheum Dis 16: 115-121, 2013.

15. de Yébenes VG, Bartolomé-Izquierdo $\mathrm{N}$ and Ramiro AR: Regulation of B-cell development and function by microRNAs. Immunol Rev 253: 25-39, 2013.

16. Hong Y, Wu J, Zhao J, Wang H, Liu Y, Chen T, Kan X, Tao Q, Shen X, Yan K and Zhai Z: miR-29b and miR-29c are involved in Toll-like receptor control of glucocorticoid-induced apoptosis in human plasmacytoid dendritic cells. PLoS One 8: e69926, 2013.

17. Monteleone K, Selvaggi C, Cacciotti G, Falasca F, Mezzaroma I, D'Ettorre G, Turriziani O, Vullo V, Antonelli G and Scagnolari C: MicroRNA-29 family expression and its relation to antiviral immune response and viro-immunological markers in HIV-1-infected patients. BMC Infect Dis 15: 51, 2015.

18. Sun XJ, Liu BY, Yan S, Jiang TH, Cheng HQ, Jiang HS, Jiang HS Cao Y and Mao AW: MicroRNA-29a promotes pancreatic cancer growth by inhibiting tristetraprolin. Cell Physiol Biochem 37: 707-718, 2015.

19. Liston A, Papadopoulou AS, Danso-Abeam D and Dooley J: MicroRNA-29 in the adaptive immune system: Setting the threshold. Cell Mol Life Sci 69: 3533-3541, 2012.

20. Wang X, Zhang C, Wu Z, Chen Y and Shi W: CircIBTK inhibits DNA demethylation and activation of AKT signaling pathway via miR-29b in peripheral blood mononuclear cells in systemic lupus erythematosus. Arthritis Res Ther 20: 118, 2018.
21. Ahluwalia JK, Khan SZ, Soni K, Rawat P, Gupta A, Hariharan M, Scaria V, Lalwani M, Pillai B, Mitra D and Brahmachari SK: Human cellular microRNA hsa-miR-29a interferes with viral nef protein expression and HIV-1 replication. Retrovirology 5: 117, 2008.

22. Hochberg MC: Updating the American college of rheumatology revised criteria for the classification of systemic lupus erythematosus. Arthritis Rheum 40: 1725, 1997.

23. Maugeri M, Barbagallo D, Barbagallo C, Banelli B, Di Mauro S, Purrello F, Magro G, Ragusa M, Di Pietro C, Romani M and Purrello M: Altered expression of miRNAs and methylation of their promoters are correlated in neuroblastoma. Oncotarget 7: 83330-83341, 2016.

24. Livak KJ and Schmittgen TD: Analysis of relative gene expression data using real-time quantitative PCR and the 2(-Delta Delta C(T)) method. Methods 25: 402-408, 2001.

25. Lipsky PE: Systemic lupus erythematosus: An autoimmune disease of B cell hyperactivity. Nat Immunol 2: 764-766, 2001.

26. Sherer Y, Gorstein A, Fritzler MJ and Shoenfeld Y: Autoantibody explosion in systemic lupus erythematosus: More than 100 different antibodies found in SLE patients. Semin Arthritis Rheum 34: 501-537, 2004

27. Zhao M, Liu Q, Liang G, Wang L, Luo S, Tang Q, Zhao H, Su Y, Yung S, Chan TM and Lu Q: E4BP4 overexpression: A protective mechanism in CD4+ T cells from SLE patients. J Autoimmun 41: 152-160, 2013.

28. Zhao JJ, Lin J, Lwin T, Yang H, Guo J, Kong W, Dessureault S, Moscinski LC, Rezania D, Dalton WS, et al: MicroRNA expression profile and identification of miR-29 as a prognostic marker and pathogenetic factor by targeting CDK6 in mantle cell lymphoma. Blood 115: 2630-2639, 2010.

29. Liu M, Zeng X, Lu YX, Mo YJ, Liao TH, Gan C and Lu XQ: Study on molecular mechanism of MiRNA-29a in promoting proliferation and invasion of non-small-cell lung cancer by inhibiting MTSS1. Eur Rev Med Pharmacol Sci 22: 5531-5538, 2018.

30. Aiello FB, Guszczynski T, Li W, Hixon JA, Jiang Q, Hodge DL, Massignan T, Di Lisio C, Merchant A, Procopio AD, et al: IL-7-induced phosphorylation of the adaptor Crk-like and other targets. Cell Signal 47: 131-141, 2018.

31. Jankowski A, Zhu P and Marshall JG: Capture of an activated receptor complex from the surface of live cells by affinity receptor chromatography. Anal Biochem 380: 235-248, 2008.

This work is licensed under a Creative Commons Attribution-NonCommercial-NoDerivatives 4.0 International (CC BY-NC-ND 4.0) License. 